\title{
Genetic counselling in the era of genomic medicine
}

\author{
As we move towards personalised medicine, it becomes more important to \\ help patients understand genetic tests and make complex decisions about their \\ health • by Jon Weil
}

$\mathrm{B}_{\mathrm{i}}$ caused concern and anxiety throu mankind's existence. Plentiful historical and contemporary examples involve the stigmatisation of affected individuals and families. Many cultures have practices and taboos that have arisen from the desire to explain and prevent such disorders. Modern science brings a growing wealth of knowledge and techniques to the diagnosis, treatment and prevention of these disorders. However, a severe birth defect or an inherited genetic disorder still creates a heavy emotional, social and financial burden not only for those directly affected, but also for their families. And while modern research has made large strides towards identifying many diseasecausing alleles, patients' fears and anxieties about their future and the future of their children remain. This is not limited to classical inherited disorders. As researchers uncover the genetic components of multifactorial diseases, many more people will be found to have mutations that put them and probably their children at risk.

The diagnosis of a genetic disorder in a newborn child, the identification of a mutation conferring an elevated risk of cancer, a positive result from a prenatal genetic screen for Down syndrome-all these scenarios confront patients with complex information to be understood and assimilated, and with an array of conflicting emotions. They require difficult decisions that involve many technical, ethical and highly personal questions (Weil, 2000). Genetic counselling can help in this process by offering various services within a clinical setting. These professionals assist patients in addressing the scientific and emotional issues that arise in such situations and help them make informed decisions based on their own values and individual circumstances (Walker, 1998).

Genetic counselling, along with many other aspects of medicine and health care, must keep pace with radical new developments in biomedical research. Building on the Human Genome Project, scientists are advancing our knowledge of the genetic contribution to common adult onset diseases, such as diabetes, different types of cancer, and cardiovascular, neurodegenerative and psychiatric disorders.

\section{A severe birth defect or an inherited genetic disorder still creates a heavy emotional, social and financial burden}

Research in pharmacogenetics is identifying genetic differences among individuals that affect their response to different medications, thus influencing both the choice and dosage of medication. At the same time, the technology to perform relatively inexpensive genetic tests for a large number of genes is expanding rapidly. These developments will, in turn, lead to tailoring risk assessment, health management and medical treatment to the individual patient. Techniques of this sort are already used in the treatment of some cancers, cardiovascular disease and neurological disorders (Collins and McKusick, 2001).

This information and technology will introduce genetic tests into many branches of medicine, leading to what has been called 'genomic medicine'. This will require that patients and the public at large understand the concepts of genetics and the information necessary to make complex decisions that are relevant to a wide variety of reproductive issues, medical interventions and lifestyle changes. Stated differently, the cognitive and emotional impact of genetics will increase dramatically in terms of its role in medicine and health care.

In most industrialised countries, genetic counselling is now provided as a component of clinical genetics, although the nature of the services and the professionals involvedmedical geneticists, genetic counsellors, nurses, social workers-varies. This article draws primarily on the perspective of genetic counselling in the USA, based on the body of theory and practice this profession has developed over the last 30 years. Genetic counsellors are trained in Masters degree programmes accredited by the American Board of Genetic Counseling (ABGC) and are certified by the ABGC. The National Society of Genetic Counselors, now in its 23rd year, represents and promotes the profession through conventions, continuing education, a code of ethics, outreach to other professions and agencies, testimony to government agencies and publication of the Journal of Genetic Counseling. Canada, and to a lesser extent Australia, New Zealand and the United Kingdom, follow a similar model of providing genetic counselling by trained specialists (Biesecker and Marteau, 1999).

Most genetic counsellors in the USA work in hospitals and university medical centres in prenatal, paediatric and general genetics. However, there are many other settings, including specialty clinics, private genetic laboratories and public health agencies. Recently, genetic counsellors 


\section{viewpoint}

have had a major role in developing services in cancer risk clinics, which is a harbinger of the expansion of medical genetics and genetic counselling into the domain of common adult onset disorders (Bernhardt et al., 1997).

In working with patients, genetic counsellors address both informational and emotional issues. They explain, in a manner consistent with the knowledge, education, interests and needs of the individual patient, the nature and limitations of genetic tests, the aetiology and clinical consequences of genetic diseases and the relevant medical and health care options. They also identify and address the complex emotions that often arise, and provide support and non-directive guidance during decision making (Baker et al., 1998). The informational and psychosocial aspects of genetic counselling are often interrelated. For example, a patient's anxiety about a forthcoming prenatal test may interfere with her ability to understand and utilise the technical
Given these critically important goals and the increasing role of genetics in medical practice, it is essential that genetic counselling be included as an

responsibility or guilt. Members of the extended family may also be at risk, or at risk of having affected children. Relatively common disorders, such as cancer or cardiovascular disease, may affect several family members due to varying combinations of genetic aetiology, environmental factors and random statistical occurrence. This can lead to beliefs that the family is 'ill-fated' or 'cursed', or that certain physical or personality traits are correlated with the disorder. Such notions have their roots in family stories, lay genetic beliefs, correct and misunderstood genetic information and family dynamics such as guilt, shame, allegiances and schisms. The decision whether to test for predisposition alleles,

integral component of health care as it evolves into the era of genomic medicine. Implementing this will involve debates and decisions concerning the level and nature of genetic counselling appropriate to different situations, the variety of professionals who will provide the services, mechanisms for financial

\section{A large number of families will have to address their genetic heritage} in order to understand and meet their health care needs

information provided during counselling. Conversely, misunderstanding or confusion about the medical implications of a test result may exacerbate feelings of anxiety or lack of control (Weil, 2000).

These genetic counselling services serve several broader goals. Genetic counsellors facilitate knowledgeable decision making that supports patient autonomy. They promote meaningful informed consent based on an adequate understanding of the technical information and its implications for the individual and his or her family. They also foster effective adjustment to difficult situations in a manner that involves a realistic assessment of the positive and negative aspects of potential outcomes, promotes individual and family competence and mobilises social and professional support-all consistent with the family's beliefs, values and culture. Last but not least, genetic counsellors promote a relationship of trust that encourages continued utilisation of their services as well as those of other health care professionals.

support and coordination with public health and public education resources. It must involve different health care professionals, legislatures and government agencies at many levels, educational institutions, insurance providers, employers and private agencies. Furthermore, all of this will be greatly influenced by the health care system of the country involved (Greendale and Pyeritz, 2001; Guttmacher et al., 2001).

\section{'Genomic medicine' requires that patients and the public at large} understand the concepts of genetics and the information necessary to make complex decisions

During its 30 years of experience, the profession of genetic counselling has identified several important concepts that will help inform these deliberations. First, genetic disorders affect families, not just individuals. If a dominant disorder has been found or if a recessive or X-linked disorder may be passed from one generation to the next, parents often have feelings of demonstrates this under circumstances that presage future developments (Bernhardt et al., 1997). Individuals often inquire about genetic testing for cancer predisposition alleles in a state of anxiety or fear concerning their own risk, that of their children and the implications of knowing versus not knowing their genotype. Any of the possible outcomes-learning that one 


\section{viewpoint}

carries the allele or does not carry the allele, that the test results were inconclusive, or that testing is not available due to inadequate family history, information or DNA samples-may trigger both positive and negative reactions. For example, learning that one carries a BRCA-1 or -2 predisposition allele may create heightened anxiety for one's own health, a

Genetic counsellors have to develop a relationship of trust with their patient to encourage continued utilisation of the service

sense of relief at knowing one's status and the ability to undertake relevant health care interventions. It involves difficult decisions concerning the medical and social ramifications of interventions, such as prophylactic mastectomy or tamoxifen therapy, guilt at the possibility that the allele has been passed on to one's children and satisfaction that one has taken a courageous step that will help one's children meet their own health care needs. Each of these factors may also affect other family members in a variety of ways (Kelly, 1991).

The preceding example was intentionally selected to illustrate one end of the spectrum of complexity associated with genetic testing. Identifying the counselling requirements of different genetic tests in a manner that includes psychosocial as well as technical characteristics and allows flexibility for the circumstances of the individual patient and family is critical (Burke et al., 2001). Within such a framework, it is essential to provide adequate counselling before, during and after the test itself.

Thirdly, decision making is a complex multi-step process influenced not only by technical knowledge but also by emotions and personal experience. The perceived burdens and benefits of the possible outcome, the extent of internal and external resources and personality characteristics such as optimism or pessimism greatly influence the interpretation of a given 'risk' figure. Faced with the uncertainty involved in a single 'roll of the dice', such as the outcome of a desired pregnancy, individuals commonly reduce numeric risk figures to a binary, non-numeric concept: 'It either will happen or it won't'. Although this reduces the information

content, it helps focus thoughts and feelings on the implications of the possible outcomes.

Given these complexities, effective decision making requires accurate information and understanding of the probabilities involved and the variability that can occur within a given diagnosis, such as Down syndrome or cardiovascular disease. It depends in part on a realistic and creative assessment of relevant resources and may require assistance dealing with emotions, such as anxiety or confusion that can cloud thinking or lead to a premature decision. When, as is often the case, more than one individual is involved, additional assistance may be needed in dealing with disagreement, conflict or difficulties in communication within the family (Weil, 2000).

Fourthly, a host of cultural, social, ethical, religious or spiritual issues influence responses and decisions. For example, individuals making reproductive decisions struggle with questions about the morality of abortion, the quality and meaning of life under differing circumstances of physical and mental ability and

Faced with uncertainty such as the outcome of a desired pregnancy, individuals commonly reduce numeric risk figures to a binary concept: 'It either will happen or it won't'

the introduction of medical technology into the personal domains of family and reproduction (Beeson and Doksum, 2001). Some families of individuals with disabilities find new and enriching aspects of life through their experiences and a changed perspective about the meaning of life. The disability rights community has presented forceful arguments to view genetic disorders and disabilities in terms of the totality of lives lived and the social barriers to achievement, not simply through a medical model comprised primarily of symptoms and limitations (Kaplan, 1993). To these concerns are added issues of privacy, confidentiality and discrimination due to the proliferation and power of computerised research and health care databases (Breithaupt, 2001). These issues will expand as genetics impinges upon more aspects of life and health care.

Fifthly, the multitude of beliefs, values, perceptions, customs, practices, family structures and institutions that influence how individuals and families respond are profoundly affected by culture and ethnicity. These include the degree of openness in discussing family history and health problems in a medical setting, the way in which different types of mental and physical disabilities are viewed in terms of their impact on individuals and families, and barriers to receiving services such as language and health care beliefs at variance with Western medicine. In order to provide appropriate and useful services, genetic counsellors thus must have information and experience about the beliefs and practices of the ethnocultural groups they serve. Equally important, however, is sensitivity to cross-cultural interactions, achieved in part through thoughtful exploration of one's own ethnocultural identity and heritage (Cohen et al., 1998; Weil, 2000).

Finally, there is the overarching issue of the relationship between the medicalgenetic and the psychosocial-counselling aspects of genetic counselling. The mechanisms of genetic transmission and their associated probabilities are precise, even when information is incomplete. The growing understanding of the human genome and the myriad of interactions involved in development and in response to environmental factors represents an ongoing scientific and technological accomplishment, as do the increasingly sophisticated techniques of clinical genetics. There is thus both reason and motivation to discuss with patients the factual, technical side of clinical genetics and its interventions. This is essential to informed decision making and adaptation to difficult situations (Smith, 1998).

\section{A host of cultural, social, ethical, religious or spiritual issues influence responses and decisions}

However, the responses of individuals and families also involve hopes, fears, imagination, adaptive mechanisms and constraining psychological defences such as denial or procrastination. Effective decision making requires thoughtful, reasonably non-defensive consideration of the meaning and impact of the various 


\section{viewpoint}

possible outcomes. Adaptation may involve finding creative new approaches to specific problems, dealing with anxiety or depression and seeing the positive as well as the negative aspects of a new and unexpected situation. It is thus essential that patients' feelings and emotional strengths and weaknesses be assessed and addressed in a manner appropriate to the-usually short-term-interventions of clinical genetics and genetic counselling (Weil, 2000).

These principles and practices of genetic counselling have built on and been augmented by contributions from other professions. Adapting these approaches to the era of genomic medicine will require careful thought, planning and attention to the many areas in which genetics will make a contribution. Clearly, the full array of evaluation and intervention described above will be neither necessary nor possible in many settings. There are also limitations of time, manpower, training and financial resources. However, as the role of genetics in medicine expands, so will the extent to which patients have to confront their genetic heritage and thus the need for appropriate services. Genomic medicine holds great promises in enhancing the quality of life for many patients but it must not be reduced to the technical aspects alone. Genetic counsellors will play an increasingly important role in this development by identifying needs, developing efficient, knowledge-based methods for appropriate services, training other health care professionals, and providing direct clinical services.

\section{Acknowledgements}

I would like to thank Margie Goldstein for suggestions and discussion while I was writing this viewpoint and for a thoughtful review of the manuscript.

\section{References}

Baker, D.L., Schuette, J.L. and Uhlmann, W.R. (1998) A Guide to Genetic Counseling. WileyLiss, New York, NY.

Beeson, D. and Doksum, T. (2001) Family values and resistance to genetic counseling. In Hoffmaster, B. (ed.), Bioethics in Context. Cambridge University Press, Cambridge, UK, pp. 153-179.

Bernhardt, B.A., Geller, G., Strauss, M., Helzlsouer, K.J., Stefanek, M., Wilcox, P.M. and Holtzman, N.A. (1997) Toward a model informed consent process for BRCA1 testing: a qualitative assessment of women's attitudes. J. Genet. Couns., 6, 207-222.

Biesecker, B.B. and Marteau, T.M. (1999) The future of genetic counseling: an international perspective. Nat. Genet., 22, 133-137.

Breithaupt, H. (2001) The future of medicine. EMBO rep., 2, 465-467.

Burke, W., Pinsky, L.E. and Press, N.A. (2001) Categorizing genetic tests to identify their ethical, legal, and social implications. Am. J. Med. Genet., 106, 233-240.

Cohen, L.H., Fine, B.A. and Pergament, E. (1998) An assessment of ethnocultural beliefs regarding the causes of birth defects and genetic disorders. J. Genet. Couns., 7, 15-29.

Collins, F.S. and McKusick, V.A. (2001) Implications of the Human Genome Project for medical science. J. Am. Med. Assoc., 285, 540-544.
Greendale, K. and Pyeritz, R.E. (2001) Empowering primary care health professionals in medical genetics: how soon? how fast? how far? Am. J. Med. Genet., 106, 223-232.

Guttmacher, A.E., Jenkins, J. and Uhlmann, W.R. (2001) Genomic medicine: who will practice it? A call to open arms. Am. J. Med. Genet., 106, 216-222.

Kaplan, D. (1993) Prenatal screening and its impact on persons with disabilities. Clin. Obstet. Gynecol., 36, 605-612.

Kelly, P.T. (1991) Understanding Breast Cancer Risk. Temple University Press, Philadelphia, PA.

Smith, A.C.M. (1998) Patient education. In Baker, D.L., Schuette, J.L. and Uhlmann, W.R. (eds), A Guide to Genetic Counseling. Wiley-Liss, New York, NY, pp. 99-121.

Walker, A.P. (1998) The practice of genetic counseling. In Baker, D.L., Schuette, J.L. and Uhlmann, W.R. (eds), A Guide to Genetic Counseling. Wiley-Liss, New York, NY, pp. 1-20.

Weil, J. (2000) Psychosocial Genetic Counseling. Oxford University Press, New York, NY.

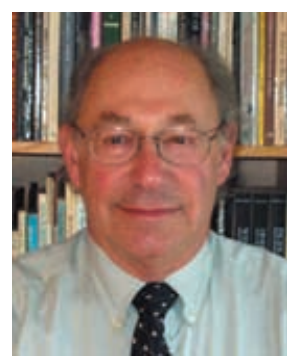

Jon Weil is the former Director of the Program in Genetic Counseling, University of California, Berkeley, CA.

E-mail: weilj@socrates.berkeley.edu

DOI: $10.1093 /$ embo-reports/kvf144 